\title{
ASPECTS DE LA DYNAMIQUE DES POPULATIONS CHEZ UN ISOPODE INTERSTITIEL
}

\author{
par \\ NICOLE COINEAU \\ Laboratoire Arago, F-66650 Banyuls-sur-Mer, France
}

\begin{abstract}
A study of the breeding cycle and population structure of Angeliera phreaticola (Isopoda, Asellota, Microparasellidae) has been carried out in the western Mediterranean. The species shows a seasonal reproductive cycle. The breeding season occurs from mid-April to the end of September. Release of juveniles is limited to the period from June to the end of September. Fourty to seventy days are necessary for the embryological development which is very long, eighty days for the post-marsupial one. It is suggested that in spring the increasing temperature of the interstitial waters accelerates the maturity of the ovocyte and post-embryonic development, and causes an advance of the breeding season. Each summerborn generation reproduces next year and yields reproducing animals two years after. Each female produces two broods (rarely three) per reproduction season and can get three to six descendants at most. Sex ratio of males to females is expressed as a function of the season and the size; males outnumber females. $A$. phreaticola has a maximum life span of about two years and two or three months.
\end{abstract}

\section{RESUME}

L'étude du cycle de reproduction et de la structure des populations de l'Isopode Angeliera phreaticola (Asellote - Microparasellidae) en Méditerranée occidentale montre une périodicité de l'activité reproductrice synchronisée avec les variations annuelles de la température interstitielle. La saison de reproduction s'étend de la mi-avril à la fin septembre; les jeunes naissent entre juin et la fin septembre. Le développement embryonnaire dure de 40 a 70 jours, le développement post-marsupial 80 jours environ. L'élévation de la température de l'eau interstitielle au printemps accélère la maturation de l'ovocyte et entraine l'avance de la saison de reproduction. Chaque génération née en été se reproduit l'année suivant sa naissance et fournit des reproducteurs pendant le second été suivant. Le rythme des pontes est lent: chaque femelle pond 2 fois par saison de reproduction (plus rarement 3 fois) et peut engendrer 3 à 6 individus au maximum. Le sex ratio établi en fonction de la saison et de la taille est en faveur des mâles. La durée moyenne de vie s'établit à 2 ans et 2 ou 3 mois.

\section{INTRODUCTION}

Angeliera phreaticola Chappuis \& Delamare, 1952 (Crustacea-Microparasellidae) est un Isopode interstitiel vivant en populations importantes dans les eaux des plages sableuses littorales de Méditerranée occidentale où il représente une part non négligeable de la biomasse. En raison de la découverte relativement récente de ces animaux mésopsammiques et de leur distribution relativement restreinte, peu de travaux leurs sont consacrés. Les facteurs écologiques qui régissent leur mode de vie et leur répartition dans certains secteurs de plages ont été précisés (Delamare Debouteville, 1960; Coineau, 1971). Une étude portant sur la reproduction de $A$. phreaticola, le développement embryonnaire et le développement post-embryonnaire met en évidence la condensation de ce dernier et la durée considérablement allongée de l'ensemble du développement; à ces effets caractéristiques du mode de vie interstitiel s'ajoutent d'autres adaptations aux milieux mésopsammique et souterrain, et en particulier, la réduction extrême du nombre d'oeufs: un seul oeuf par ponte est émis (Delamare Deboutteville, 1960; Coineau, 1971). Des recherches à propos de l'anatomie de ce Crustacé confirment son adaptation très poussée à la vie dans les sables: l'existence d'une seule gonade impaire chez la femelle, la simplification de l'appareil génital mâle, le tube digestif rectiligne, l'étirement en longueur des différentes structures cérébrales sont autant de traits adaptatifs remarquables (Coineau \& Renaud-Mornant, 1977; Renaud-Mornant \& Coineau, 1978).

L'étude de la structure et de l'évolution des populations naturelles de $A$. phreaticola vient compléter ces données et pourra étayer ultérieurement des travaux au sujet de la croissance, de la production et de bilans énergétiques . 


\section{MATERIEL ET METHODES}

Les Isopodes proviennent des plages littorales d'Argelès-surMer (Pyrénées-Orientales). Ils sont extraits du sable par la méthode de sondage Karaman-Chappuis dont Karaman (1933) est l'initiateur et que Chappuis (1942) a mise ensuite au point. Les prélèvements ont lieu une fois par mois, plus souvent pendant la période de reproduction, durant une année (janvier 1977 à décembre 1977) ${ }^{1}$ ). Entre 1975 et 1980 , le même rythme de récoltes a été maintenu en vue d'études différentes. Les animaux fixés sont mesurés au micromètre oculaire, au microscope, entre le bord rostral du céphalon et le bord caudal du telson, corps en extension. Les catégories suivantes peuvent être distinguées: jeunes (de stades I, II, III et IV, selon Coineau, 1971), femelles reproductrices ou femelles présentant des oostégites, femelles normales dépourvues d'oostégites, et mâles adultes.

\section{RESULTATS}

\section{Période de reproduction - recrutement}

Les oostégites sont des caractères sexuels primaires temporaires, n'apparaissant qu'au moment de la ponte et persistant pendant toute la durée du développement embryonnaire de la portée; ils

1) Tous les prélèvements ont été effectués par F. Duran, que je remercie vivement. disparaissent lors de la libération du jeune qui correspond à une mue de la mère. Les femelles reproductrices permettent donc de cerner avec précision la période de reproduction. Les variations de leurs pourcentages par rapport au nombre total des femelles adultes traduisent le taux d'activité reproductrice de la population (fig. 1).

En 1977, les premières femelles gestantes apparaissent début mai avec un taux de 40,24\%; ce dernier croît en juin, passe par un maximum en juillet et s'abaisse progressivement en août et septembre pour revenir à zéro en octobre. La période de reproduction se déroule donc entre la fin avril et la fin septembre. L'évolution saisonnière de l'appareil génital femelle suivie en 1976 par coloration in toto et coupes, montre une durée équivalente de la période de reproduction (Coineau \& Renaud-Mornant, 1977).

Les ovigères de mai-juin-juillet correspondent aux femelles nées l'été de l'année précédente $\left(G_{\imath}\right.$ de 1976) représentées par les femelles de taille réduite ou moyenne (classes 11 à 16 avec un maximum dans les classes 13 et 14), auxquelles s'ajoutent les grandes femelles nées en été $1975\left(G_{1}\right)$ des classes 16 à 20, qui se sont déjà reproduites

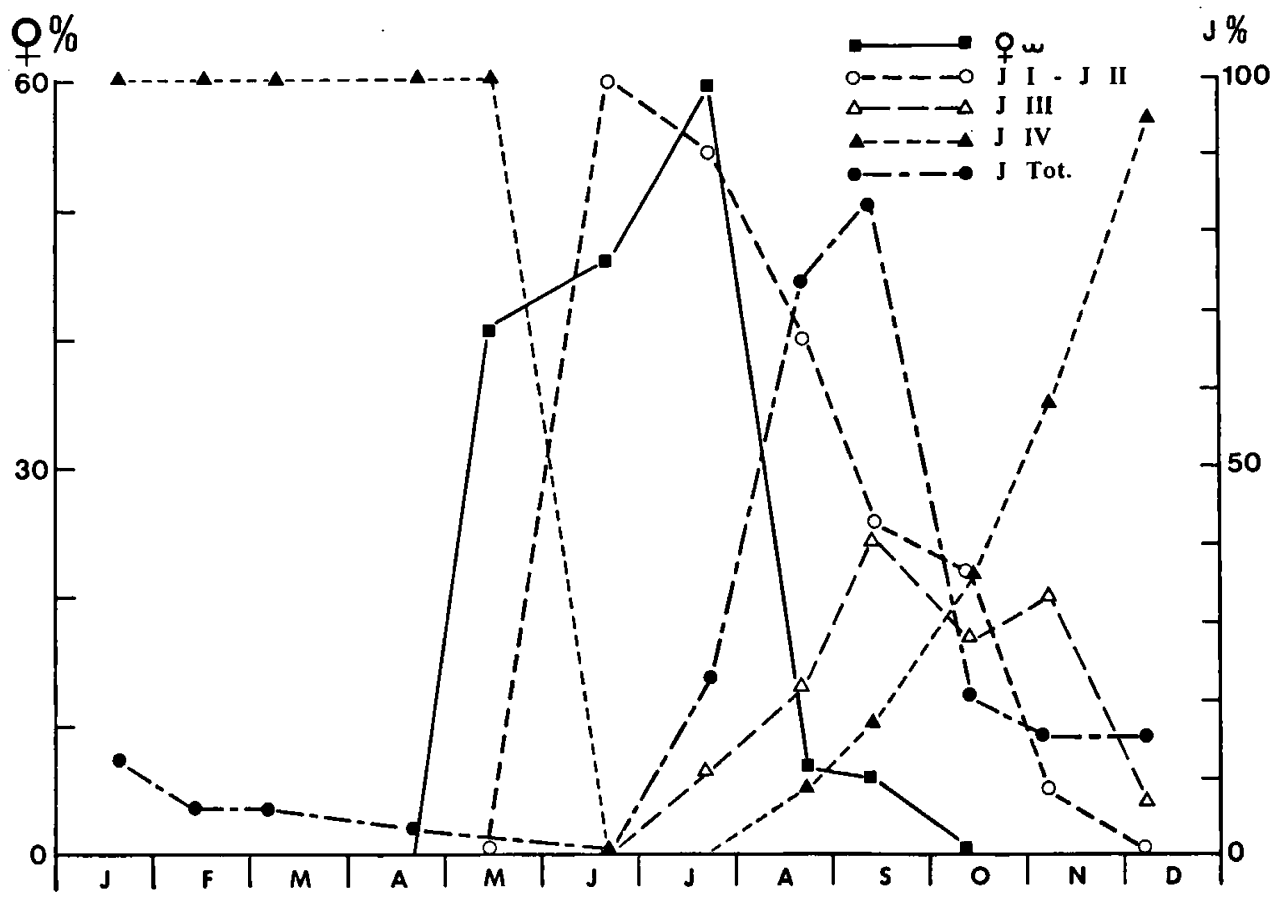

Fig. 1. Evolution du pourcentage mensuel de l'ensemble des jeunes par rapport à la population totale, des jeunes de stade I, II, III et IV par rapport à l'ensemble des jeunes, et des femelles reproductrices par rapport au total des femelles adultes. 
l'année passée. En fin juillet-août et septembre, les femelles $G_{1}$ de plus fortes tailles disparaissent et les reproductrices $G_{2}$ n'atteignent que des tailles moyennes (classes 13 à 17) et sont probablement issues des naissances de août-septembre de l'année 1976 pour les moins grandes qui se reproduisent peut-être pour la première fois, et des naissances de juin-juillet 1976 pour les plus longues.

La saison de reproduction est donc nettement limitée à la période estivale fin avril à septembre. Les individus de toutes les catégories de taille adulte de la population femelle se reproduisent au début de l'été tandis que le stock des jeunes femelles va en s'appauvrissant régulièrement d'avril à septembre (fig. 3 ).

Les premiers jeunes s'échappent du marsupium à la mi-juin en 1977 , soit un mois et demi après l'apparition des premières pontes. Le développement embryonnaire s'est poursuivi entre $12^{\circ}$ et $18^{\circ} \mathrm{C}$. En 1979 , la durée du développement intramarsupial s'établit aux alentours de 40 jours, entre $12^{\circ}$ et $15^{\circ} \mathrm{C}$; il en est de même en 1980: 45 jours environ à une température comprise entre $11,5^{\circ}$ et $14^{\circ} \mathrm{C}$ (fig. 2). En 1966, le développement embryonnaire s'est poursuivi pendant au moins 70 jours, par une température de l'eau interstitielle allant de $14^{\circ}$ à $21^{\circ} \mathrm{C}$. Il ne semble donc pas y avoir d'incidence de la température sur la durée du développement intramarsupial.

Par contre, un léger décalage de la saison de reproduction dans le temps se produit lorsque la température interstitielle s'élève rapidement au printemps: c'est le cas de l'année 1966 au cours de laquelle les premières ovigères ont été récoltées dès le 14 avril, à $14^{\circ} \mathrm{C}$; en 1967 et 1979 , elles apparaissent les 20 et 25 avril, à $12,8^{\circ}$ et $12^{\circ} \mathrm{C}$. En 1976, 77, 78 et 80, les premières femelles reproductrices ne sont recueillies qu'au début de mai entre $11,5^{\circ}$ et $12^{\circ} \mathrm{C}$. La saison de reproduction semble donc avancée lorsque la température interstitielle est plus élevée. Il est probable que la maturation de l'ovocyte se trouve accélérée par une augmentation de la température. Cette hypothèse rejoint les résultats des travaux expérimentaux de Mocquard et al., 1980, qui montrent que les longues photophases et une température plus élevée

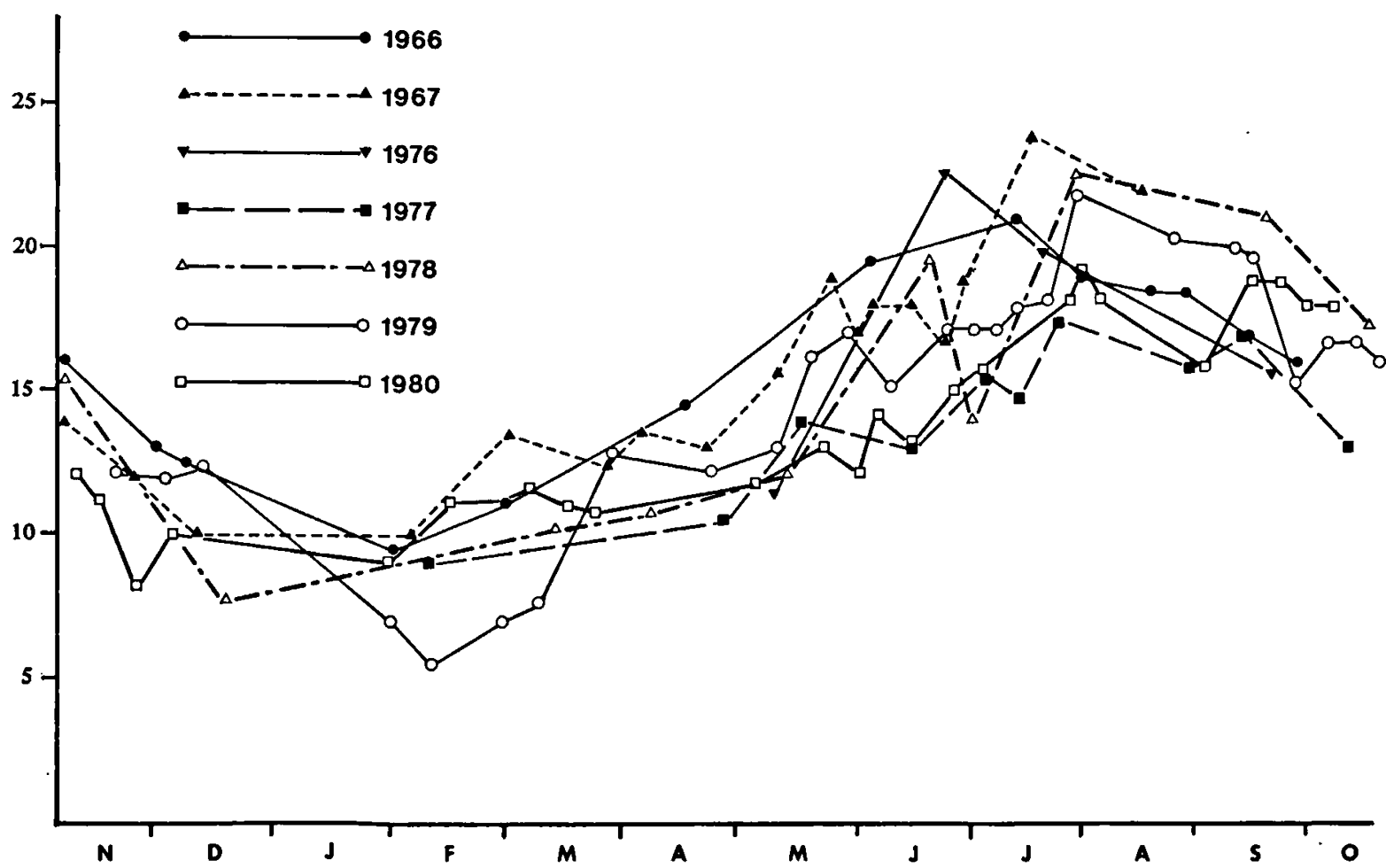

Fig. 2. Variations de la température $\left({ }^{\circ} \mathrm{C}\right)$ de l'eau interstitielle littorale à Argelès-Plage au cours des années $1966-67$ et 1976 à 1980 . 
accélèrent le déclenchement de la maturation ovarienne des Oniscoïdes. Dans le cas d'Angeliera, bien sûr, la photophase n'intervient pas, le milieu interstitiel étant obscur.

Les naissances les plus abondantes se situent en juillet, août et septembre. Les jeunes de stades I et II ont été représentés par la même courbe, le stade I étant très fugace et particulièrement fragile (fig. 1). Les courbes représentatives des jeunes I, II et III évoluent parallèlement, avec un décalage d'un peu moins d'un mois; de même, les premiers stades IV apparaissent environ 25 jours après les premiers stades III. La durée approximative de chacun des stades post-embryonnaires II, III et IV est donc voisine d'un peu moins d'un mois (environ 25 jours) en été, de sorte que la génération fille rattrape la taille des plus petits éléments de la génération mère dès la fin août (fig. 3).

L'ensemble du développement post-marsupial se déroule donc en deux mois $1 / 2$ en été. Par contre, les stades IV atteints en décembre ne deviennent que lentement adultes pendant les mois d'hiver. Tous les stades III sont devenus des stades IV en janvier. En 1966, les stades II et III réunis ne durent pas plus d'un mois en juillet-août (Coineau, 1971). Si l'on se reporte aux courbes de température de l'eau interstitielle (fig. 2) on s'aperçoit que celle-ci est plus élevée en 1966, et en 1967. Le développement post-marsupial se déroulerait donc plus rapidement à une température plus élevée.

\section{Evolution de la structure de la population au cours d'un cycle annuel (fig. 3)}

Les Angeliera sont répartis de 50 en $50 \mu \mathrm{m}$ en classes de taille, de $800 \mu \mathrm{m}$ à $1800 \mu \mathrm{m}$.

En janvier, la population se compose essentiellement d'adultes (classes 11 à 20): nous reconnaissons d'une part les individus nés en été de l'année précédente $\left(G_{2}\right.$ nés en 1976), les plus nombreux et déterminant un mode dans les classes 14 et 15 , et d'autre part les grands adultes $\left(G_{1}\right.$ nés en été 1975), moins nombreux et occupant les classes 16 à 20; il reste peu de jeunes de stade IV dans les classes 8 à 10. En février et mars les repré- sentants de la classe 8 ont disparu, ceux des classes 9 et 10 diminuent, ce qui correspond à la croissance des jeunes de stade IV. En même temps, les classes 12 et 13 gagnent des représentants. Le mode persiste dans la classe 14 seulement, ce qui s'explique par la croissance des jeunes adultes des classes précédentes. En avril, ce mode se décale vers la droite, et les jeunes de stade IV n'occupent plus qu'une classe, comme le mois suivant.

En mai, la croissance des adultes a donné de plus nombreux représentants dans les classes 15 à 19; les femelles reproductrices se situent dans toutes les classes, avec un mode dans les classes 13-14 et un autre dans la classe 16. La génération fille $G_{s}$ apparaît en juin: elle est faiblement représentée car très peu de jeunes ont éclos; tous les jeunes de $G_{2}$ sont devenus adultes, susceptibles de se reproduire en août-septembre. En juillet, la naissance de la génération fille se poursuit, apportant des représentants dans les classes 1 à 8 au fur et à mesure que la croissance se manifeste; l'ensemble de l'histogramme se déplace par roulement vers la droite pour les adultes; les femelles reproductrices montrent 2 modes (13 et 16-17) correspondant aux femelles $G_{2}$ de la fin de l'été 1976 (13) et à celles du début de l'été 1976 et de $G_{1}$ de 1975. En août, par suite de la croissance des premiers nés, les classes 8-9-10 se „garnissent", de sorte que la génération fille rattrape la génération mère en taille; les classes 18-19-20 perdent des éléments par suite du décès des plus grands adultes $G_{1}$ de 1975 .

L'histogramme de septembre permet d'observer un premier mode dans la classe 5 dû aux jeunes de stade II, un second dans la classe 7 correspondant aux jeunes de stade III, un 3ème classe 9 (jeunes de stade IV) tandis que des jeunes nés en juin donnent déjà de petits adultes formant les classes 11 et 12 qui n'existaient plus en juillet; notons que la croissance de la génération fille se déroule rapidement pendant les mois chauds d'été: la translation vers la droite sur les histogrammes correspondants s'opère plus rapidement que pendant les mois d'hiver (fin janvier-février, mars); c'est ainsi qu'en octobre de jeunes adultes atteignent les classes 13 et 14; les naissances sont terminées. En novembre, puis décembre, la croissance des derniers jeunes continue à se traduire 

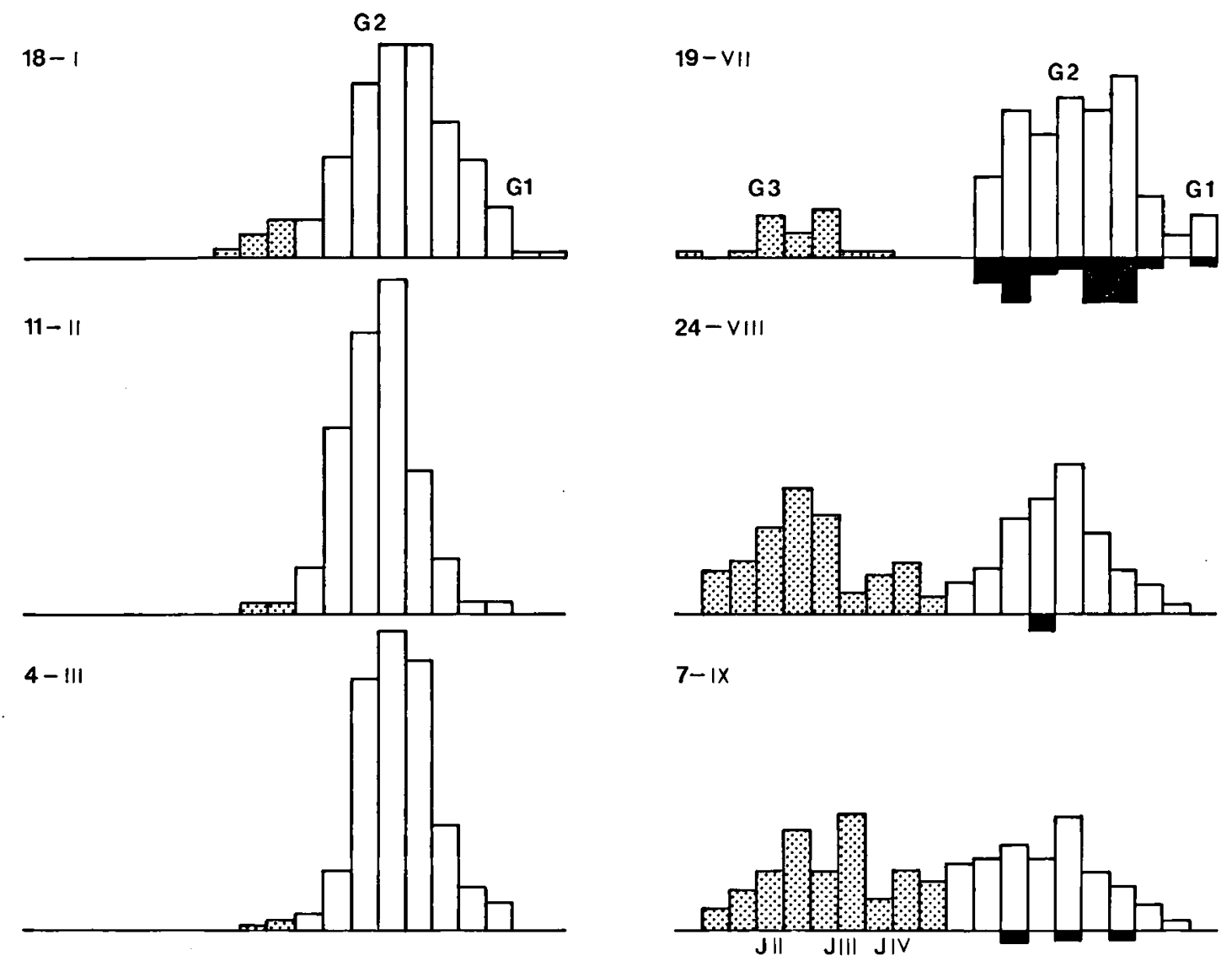

$24-$ VIII
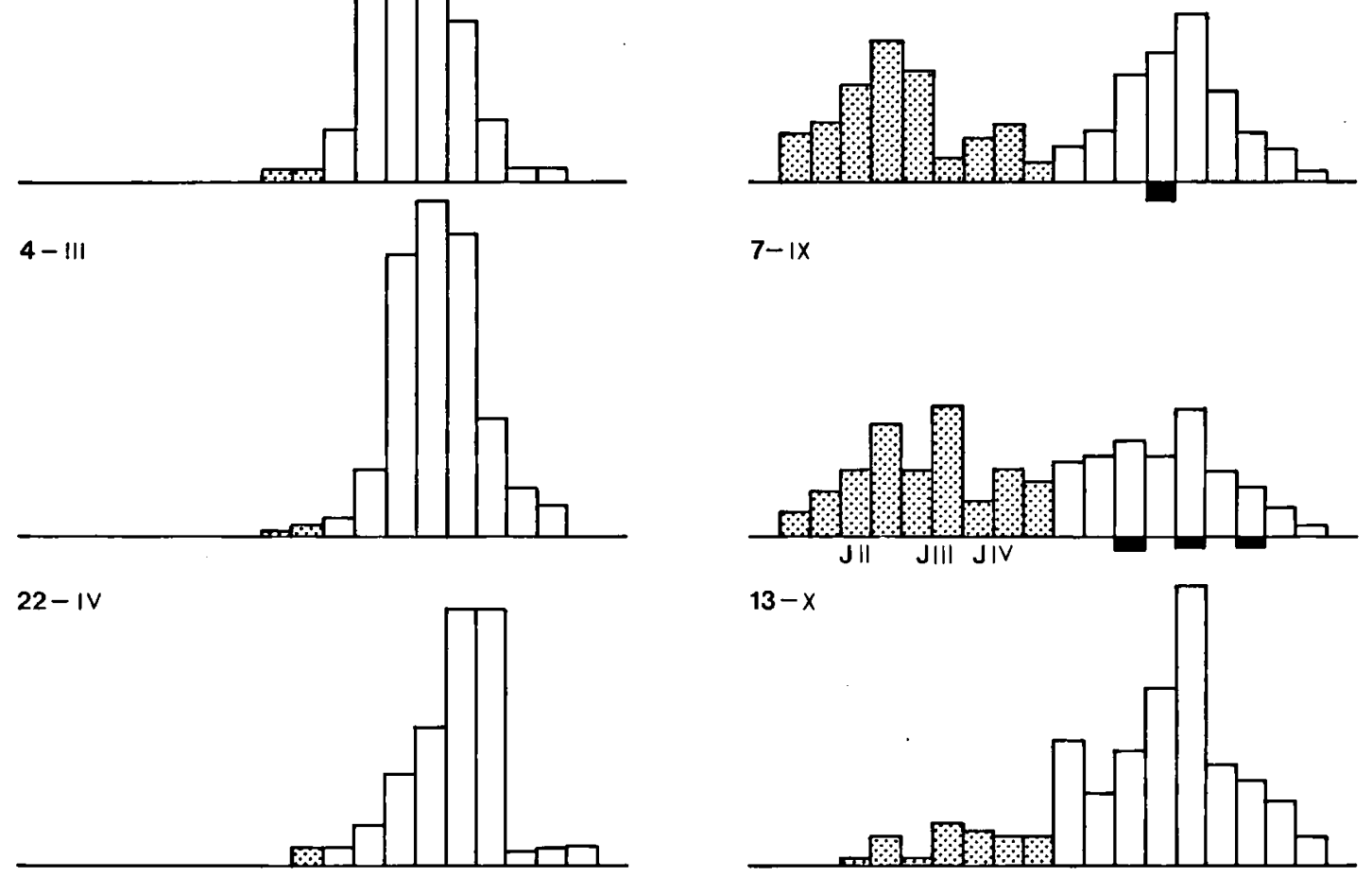

$7-1 X$

$12-V$
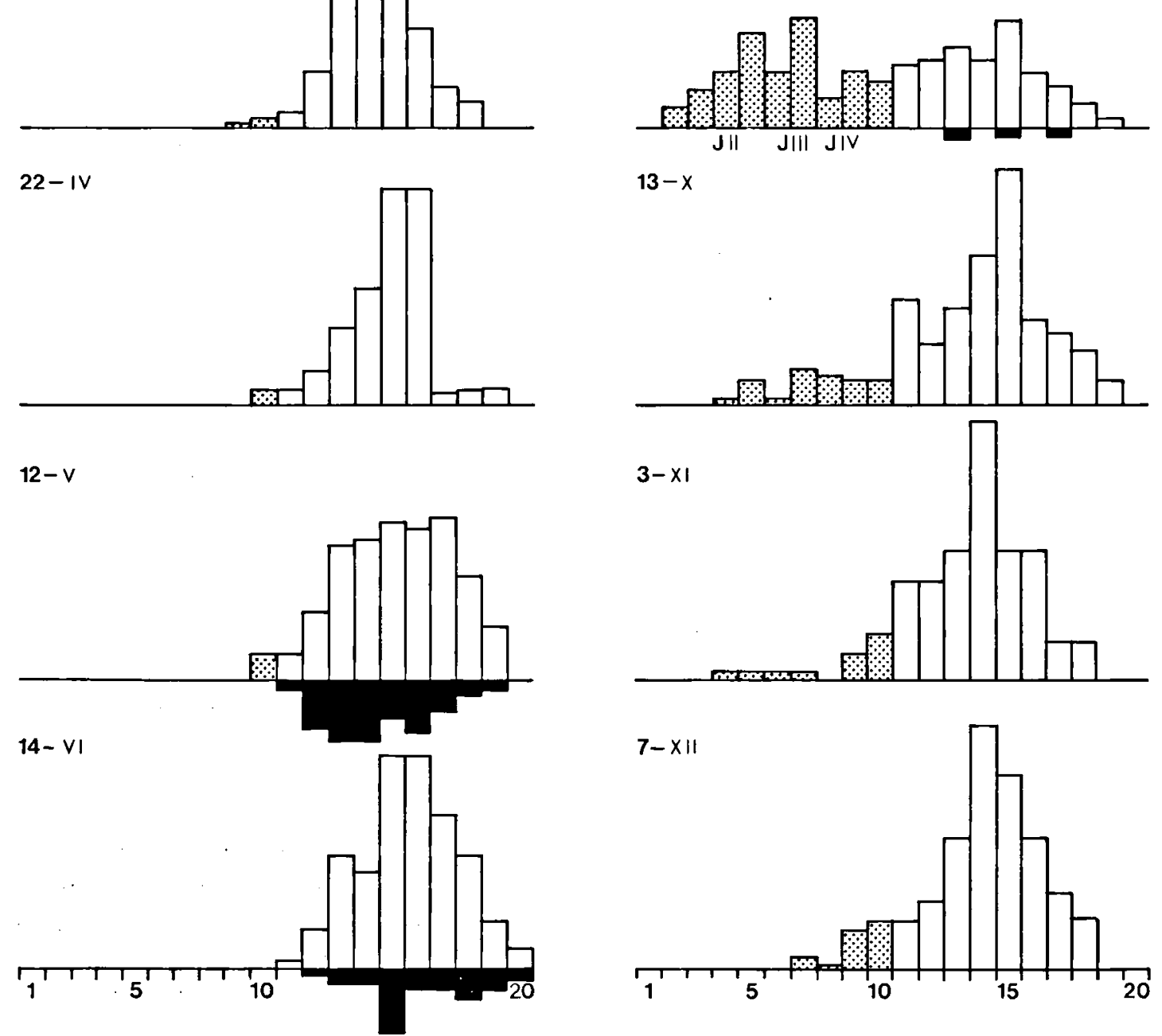

Fig. 3. Histogrammes de répartition mensuelle des tailles de Angeliera phreaticola dans la population d'Argelès-sur-Mer, année 1977. Abscisse: classes de taille; ordonnée: pourcentage de chaque classe de taille par rapport à la population totale; pointillé: jeunes; en noir: femelles reproductrices. 
par le gain de classes successives ( 7 à 10) tandis que les jeunes adultes de la génération fille donnent un mode dans les classes 14 et 15 . La génération mère $G_{z}$ occupe les classes $16-17$ et 18 , remplaçant les grands adultes de $G_{1} 1975$ disparus au cours de l'été. L'histogramme de décembre est proche de celui de janvier. Celui de janvier de l'année suivante est très semblable à celui de janvier 1977.

L'analyse par histogrammes de la répartition des tailles permet donc de suivre l'enchaînement des générations et apporte en outre des données au sujet de la croissance. La génération mère d'une année se compose des adultes issus d'une part des individus nés en été de l'année précédente, et d'autre part de ceux nés deux années avant. Une femelle peut présenter deux portées consécutives seulement par an, dans le cas général, étant données la durée du développement intramarsupial ( 40 à 70 jours) et celle de la période annuelle de reproduction ( 5 mois à 5 mois et demi). Il peut arriver que 3 pontes se succèdent au cours du même été pour une femelle ayant pondu dans la seconde quinzaine d'avril. En effet, on peut observer que la croissance de l'ovocyte suivant se poursuit pendant la durée du développement embryonnaire de la portée et qu'au moment de la mise bas, sa maturation est toute proche (Coineau $\&$ RenaudMornant, 1977). Si la température de l'eau interstitielle est élevée, cette maturation de l'ovocyte s'accélère; une seconde ponte peut alors survenir à la mi-juin, et une troisième à la mi-août.

Chaque femelle procrée l'année suivant sa naissance, ainsi que deux ans après. Quelques femelles nées début juin pourraient peut-être se reproduire dès août de la même année puisqu'elles deviennent adultes en août, la température élevée favorisant la maturation accélérée de l'ovocyte. Les histogrammes montrent en effet des femelles ovigères de petite taille en septembre (classe 13). Cette éventualité ne pourrait correspondre qu'à des femelles nées début juin.

Chaque femelle peut donc engendrer de 3 à 6 individus au maximum, puis survient la mort. Il semble que la durée moyenne de vie puisse s'établir aux environs de deux ans et quelques mois.

\section{Sex ratio (fig. 4)}

Nous entendons par sex ratio le rapport nombre de mâles sur nombre de femelles de la population adulte. Il s'équilibre à une moyenne de 1,24 pour l'année 1977. Le nombre de mâles est donc supérieur à celui des femelles. Les variations de ce rapport suivies mensuellement (fig. 4) ne sont pas négligeables. Voisin de 1 en août, il croît peu mais constamment jusqu'en janvier, passe par un maximum $(1,62)$ en mars, puis diminue régulièrement jusqu'à 0,82 en mai, seul mois pendant lequel on dénombre davantage de femelles que de mâles; remarquons qu'il s'agit du début de la période de reproduction. Le rapport numérique

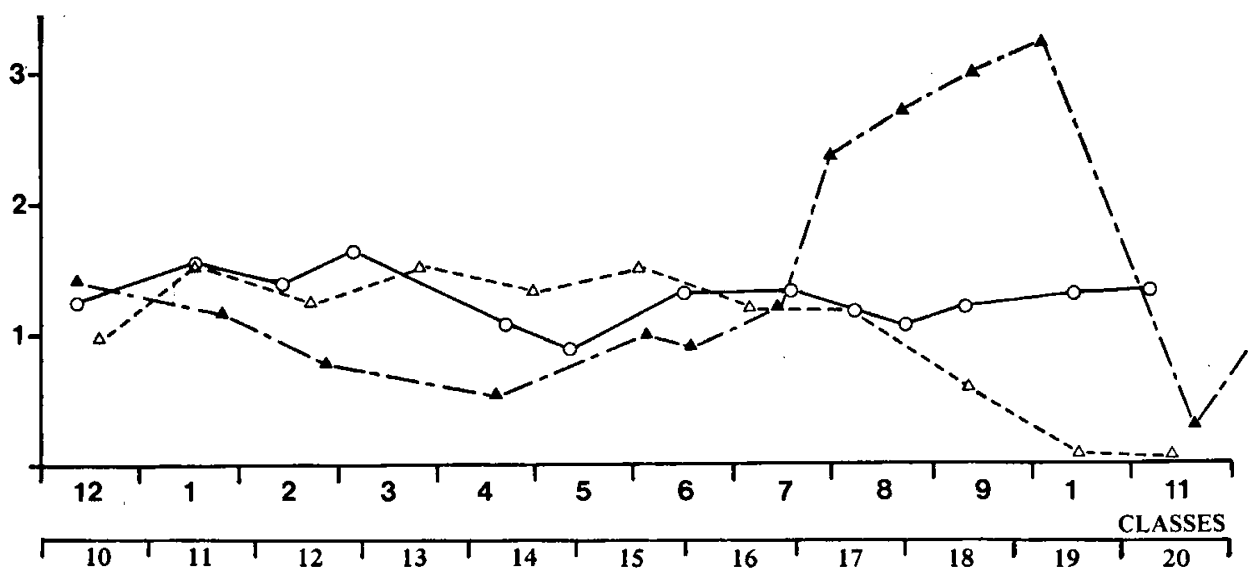

Fig. 4. Rapport numérique des sexes pendant l'année 1977. O, Angeliera phreaticola, variations mensuelles; $\Delta$, Angeliera phreaticola, variations en fonction de la taille; $\boldsymbol{\Delta}$, Microcharon marinus, variations mensuelles. 
des sexes augmente à nouveau en juin et subit une nouvelle baisse en août. Il reste donc un peu plus faible (moyenne 1,13) pendant toute la période de reproduction. Le taux de reproduction relativement bas de cette espèce (femelle ne pondant qu'un seul oeuf à la fois, deux dans le cas général par saison de reproduction) n'est donc pas compensé par un déséquilibre de la proportion des sexes en faveur des femelles. L'étude du sex ratio par classes de longueur au cours de l'année 1977 montre qu'une relation semble exister entre le sex ratio et la taille (fig. 4). Le rapport s'abaisse dans la classe 16, puis s'inverse et devient inférieur à 1 à partir de la classe 17 , tend vers zéro dans les classes 19 et 20 .

Les courbes de fréquence de taille selon le sexe (fig. 5) ainsi que celles du sex ratio par taille, établies mensuellement, permettent de mieux mettre en évidence les variations du sex ratio en fonction de la taille. Les femelles atteignent des tailles plus élevées que les mâles qui ne sont représentés qu'au mois de juillet dans la classe 20 , et très rarement dans la classe 19 pendant toute l'année. La courbe de fréquence des femelles est le plus souvent décalée vers la droite. Le sex ratio, exprimé ici en pourcentage de mâles, reste supérieur à $50 \%$ dans les classes 10 à 15 ou 16 tout en décroissant régulièrement peu à peu, de janvier à avril; il chute au-dessous de $50 \%$ à partir de la classe 17 et tombe rapidement à zéro. En mai, il demeure inférieur à 50\% dans toutes les classes de taille (exception 16). Pendant les mois d'été, le pourcentage des mâles est moins élevé dans les classes 10 à 16 et descend au-dessous de $50 \%$ à partir de la classe 18. En automne, la courbe se rapproche progressivement de celles d'hiver, avec passage au-dessous de 50\% dans la classe 17 .

Si l'on examine les proportions des sexes parmi les stades post-embryonnaires III et IV, on s'aperçoit qu'il existe davantage de femelles au stade III (30\% de mâles à ce stade), alors que l'inverse se produit au stade IV (53,6\% de mâles). Le passage du stade III au suivant semble donc critique pour les femelles qui naissent donc en plus grand nombre.

\section{DISCUSSION}

Angeliera phreaticola et Microcbaron marinus, autre Isopode interstitiel cohabitant dans les mêmes biotopes, se reproduisent simultanément; le pourcentage de femelles reproductrices est plus élevé en juin pour Microcharon, en juillet pour Angeliera. La saison de reproduction d'Angeliera débute à la mi-avril, à la fin avril ou au début de mai selon la température de l'eau interstitielle, comme celle de Microcharon (Coineau, 1971); elle s'étend jusqu'à la fin septembre. Les résultats confirment la périodicité de l'activité reproductrice des Isopodes interstitiels littoraux, vivant dans des milieux instables et soumis à des variations saisonnières de température. La saison de reproduction coïncide avec la période des témpératures les plus élevées (figs. 1 et 2). Des observations analogues ont été formulées par Ax (1977) au sujet des Turbellariés, ainsi que par Hall \& Hessler (1972) à propos du Mystacocaride Derocheilocaris typica, tandis que McLachlan (1977) conclue que Derocheilocaris algoensis se reproduit de façon continue toute l'année, comme $D$. remanei (cf. Delamare Deboutteville, 1960). Plusieurs auteurs ne constatent pas de périodicité des fonctions reproductrices: Remane (1927) chez Halammobydra, Swedmark (1955) pour Psammodrilus, Faubel (1976) pour les Turbellariés; ce dernier montre qu'il y a maturité sexuelle toute l'année, mais que l'émission des oeufs dépend de la température.

On ne peut donc pas parler d'un comportement reproducteur typique des animaux interstitiels, alors qu'il était admis, il y a une vingtaine d'années que ceux-ci se reproduisaient toute l'année (Remane, 1959; Delamare Deboutteville, 1960). Il semble bien que l'absence de périodicité soit plutôt caractéristique des animaux souterrains continentaux vivant dans des milieux écologiquement plus stables (température en particulier); et encore ne s'agit-il pas d'un phénomène quasi général. Ainsi Lescher-Moutoué (1974) constate que certains Cyclopides (comme Speocyclops) se reproduisent toute l'année mais avec un maximum d'activité en automne et au printemps. Magniez (1976b) pense qu'une „saison de ponte semble exister chez Stenasellus virei" et que la richesse en nourriture du biotope représente un facteur 

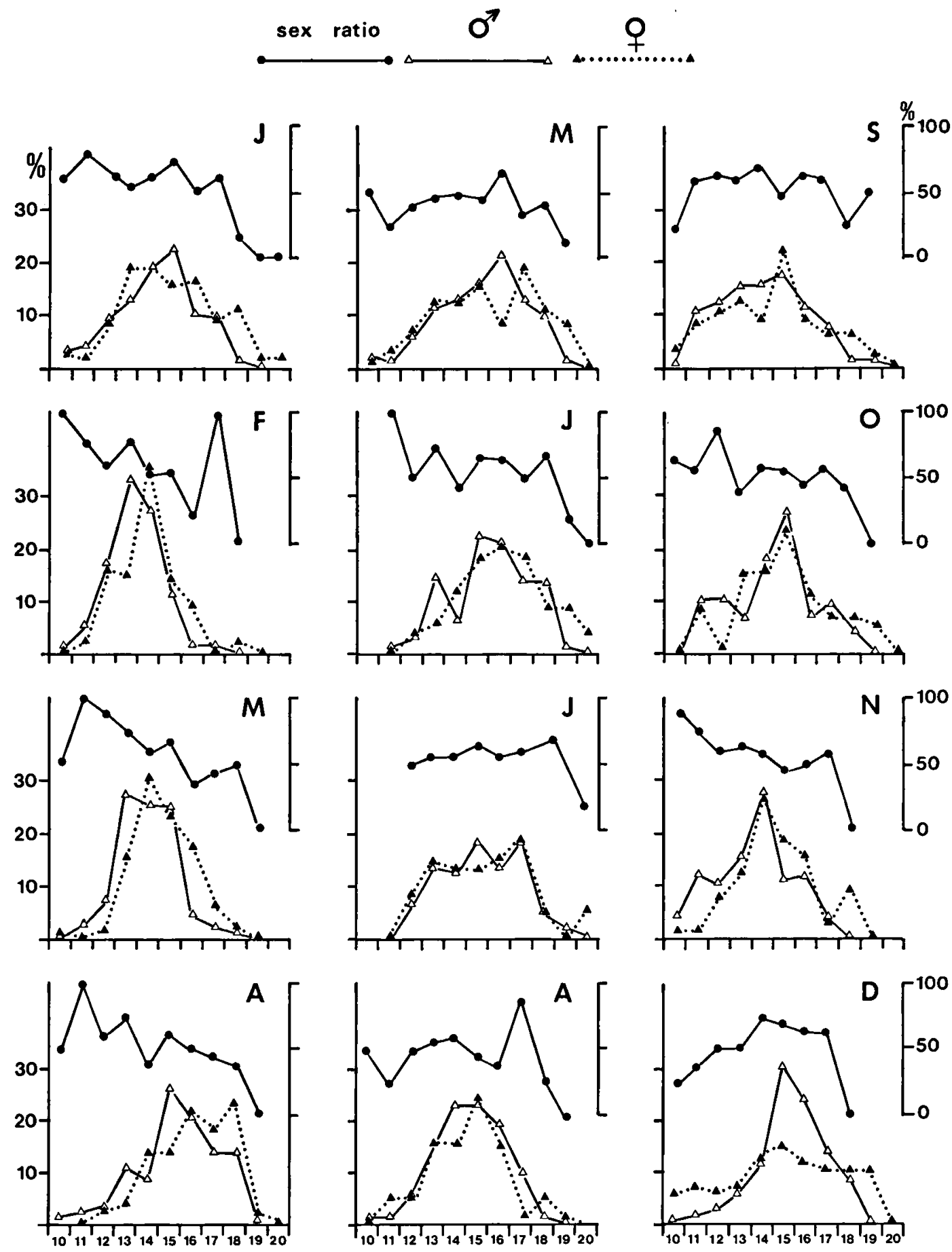

Fig. 5. Courbes de fréquence de taille mensuelles des mâles et des femelles et sex ratio établi en pourcentages mensuels des mâles par rapport à l'ensemble des adultes (année 1977). 
important. De même, Ginet (1969) montre que Niphargus se reproduit tout au long de l'année, mais présente un rythme annuel „saisonnier influencé par la qualité de l'eau souterraine en tant que véhicule de nourriture et d'oligo-éléments nécessaires à la physiologie sexuelle de ces Amphipodes". Par contre, chez certains Copépodes harpacticides (Nitocrella subterranea, Bryocamptus pyrenaicus ... Rouch (1968) ne décèle aucune variation saisonnière. Il en est de même pour certaines Bathynelles (Jakobi, 1954).

Une activité reproductrice continue chez des représentants psammiques littoraux ou benthiques marins représente peut-être le témoignage d'une adaptation plus poussée au milieu interstitiel.

Chez l'Isopode Asellote Jaera dont Angeliera est relativement proche, Jones \& Naylor (1971), Jones (1974), et Pantoustier \& Prunus (1977) montrent que différentes espèces de ce genre se reproduisent toute l'année avec un maximum plus marqué au printemps et en été.

Le rythme des pontes est particulièrement lent chez Angeliera, comme chez Microcharon. Deux pontes seulement sont possibles à une femelle de Microcharon au cours d'une saison de reproduction. Une cadence d'une ponte tous les 50 jours peut avoir lieu chez.Angeliera soit un maximum de 3 pontes d'un seul oeuf de très forte taille par été, les années les plus chaudes; 2 pontes seulement par période estivale se produisent plus généralement. Il faut noter ici le rôle de la température qui agit sur la vitesse de maturation de l'ovocyte, entraînant ainsi l'avance ou le retard de la période de reproduction. Un tel rythme contraste avec celui du genre Jaera qui pond toutes les trois semaines (Bocquet, 1953). Par contre, les pontes sont très espacées chez les Crustacés souterrains comme Sienasellus virei ( 1 ponte tous les deux ans ou même tous les 5 ans, en raison de la longue durée de l'intermue de reproduction, Magniez, 1976a) ou comme les Copépodes harpacticoïdes (Rouch, 1968).

L'ensemble du développement de Angeliera se déroule en 4 mois, alors que celui de Microcharon dure 5 mois. La différence vient de l'allongement de la phase intramarsupiale chez Microcbaron (2 mois). Le développement post-marsupial s'accom- plit plus rapidement chez Angeliera puisque 4 stades conduisent le jeune de la naissance à l'adulte en 2 mois $1 / 2$, alors que 3 stades seulement sont nécessaires à Microcharon pendant 3 mois. Ces données ne sont valables que pour l'été car en hiver, la croissance des stades post-embryonnaires se trouve ralentie.

Si la température ne semble pas influencer la durée du développement embryonnaire, elle agit sur la vitesse de maturation de l'ovocyte, qu'elle accélère, et sans doute aussi sur le déclenchement de celle-ci à la fin de l'hiver. Si l'on examine les courbes de température de 1966, 1977 et 1979, il semblerait que ce soit plutôt l'augmentation rapide de la température qui accélère la vitesse de maturation, plutôt qu'une température plus régulière, ou n'augmentant que peu à la fois. De March, 1978, constate d'ailleurs que „la durée de la maturation complète et la durée du développement embryonnaire sont inversement proportionnels à la température chez des animaux gardés à des températures constantes. La durée de la maturation est plus longue que prévue dans le cas où le développement des premiers stades se fait à de basses températures et plus courte que prévue si le développement des premiers stades se fait à des températures plus élevées". Hunt \& Robertson (1977) montrent que la vitesse de développement croît avec l'augmentation de la température chez le Copépode Cyclops. Lescher-Moutoué (1974) formule des résultats analogues chez Speocyclops. La plupart des auteurs mentionnent que la saison de reproduction des animaux interstitiels manifestant une périodicité des fonctions reproductrices se place pendant les périodes chaudes. Par des séries d'expériences sur divers Isopodes terrestres, Mocquard et al. (1976, 1978 et 1980) montrent que l'induction et la durée de la période de reproduction des Oniscoïdes sont contrôlées par des facteurs externes comme la température et la photopériode, et que le repos sexuel est „assuré dans les conditions naturelles par les basses températures et les jours courts"; l'élévation de la température accélère le processus d'entrée en activité en entraînant une augmentation du rythme des mues.

L'étude de la structure de la population montre que, chaque année, comme chez Microcharon marinus, la génération mère du printemps com- 
prend des adultes provenant des naissances de l'été précédent et de ceux nés 2 années plus tôt; chaque génération fille se reproduit seulement l'année suivant sa naissance entre avril et octobre, ainsi que la seconde année après sa naissance au début de la saison de reproduction. Une femelle de Microcharon ne peut engendrer que 2 à 4 descendants, alors que chez Angeliera une femelle peut engendrer 3 à 6 individus lorsque la température interstitielle est élevée dès le début du printemps.

Le rapport numérique des sexes, globalement sur une année, est plus élevé chez Microcharon marinus (1,54) que chez Angeliera (fig. 4). Ses fluctuations mensuelles sont nettement plus accentuées que pour Angeliera; les mâles sont beaucoup plus nombreux en août, septembre et octobre, ce qui semble indiquer qu'une certaine proportion de grandes femelles de Microcharon disparaissent après leur seconde mise-bas en fin de saison de reproduction, ce phénomène étant bien moins accusé chez Angeliera; la prédominance des mâles est permanente (mai excepté pour Angeliera) alors qu'une tendance inverse se remarque pour Microcharon un peu avant la saison de reproduction. La tendance à l'équilibre des sexes au moment de la saison de reproduction qui se manifeste souvent chez d'autres Crustacés et qui permet de mieux assurer le potentiel reproducteur (Jones, 1974; Daguerre de Hureaux, 1979; Louis, 1977) ne se réalise dans les deux cas étudiés ici que de façon très atténuée et ponctuelle (juin, Microcharon; mai, Angeliera). Tout au plus constate-t-on une baisse du rapport mâles/femelles pendant la période de préparation à la reproduction.

Janssen et al. (1979) et Kinne (1952) invoquent l'influence de la température sur la détermination du sexe et le sex ratio de certains Amphipodes du genre Gammarus. Pour Wenner, 1972, les exemples de déviations du sex ratio sont fréquents ches les Crustacés, et les variations de celui-ci en fonction de la taille sont plus cohérentes que les variations globales mensuelles généralement calculées. L'auteur différencie ainsi quatre types de relation sex ratio/taille: standard, d'inversion, intermédiaire et anormal. Garcia \& Albaret (1977) utilisent cette relation avec la taille pour décomposer par sexe les histogrammes de fréquence de tailles, afin de pouvoir travailler sur des grands nombres.

Les courbes de sex ratio en fonction de la taille pour Angeliera sont délicates à interpréter, mais montrent que effectivement, le sex ratio dépend de la taille, particulièrement dans les classes dc rang élevé. Mais il est évident que plusieurs phénomènes peuvent intervenir simultanément, en plus de différences liées au sexe, tels que la croissance différentielle, des migrations et la mortalité (à la fin de la saison de reproduction).

Cette étude souligne la lenteur du cycle reproducteur des Isopodes interstitiels. Angeliera phreaticola représente un cas limite, au taux de reproduction extrêmement bas: chaque femelle qui ne possède qu'une seule gonade, n'émet qu'un oeuf par ponte, et n'assure que deux cycles gonotrophiques par saison de reproduction, pendant deux années consécutives. Cependant, un potentiel reproducteur si peu élevé suffit à maintenir l'équilibre démographique car le milieu interstitiel est un milieu conservateur dans lequel la concurrence vitale est limitée, les prédateurs étant relativement rares. Il faut noter aussi la longue protection assurée aux jeunes pendant toute la durée du développement embryonnaire qui se déroule très longuement à l'intérieur de la cavité marsupiale de la mère, ainsi que la condensation du développement post-embryonnaire. De telles modalités rejoignent des tendances communes aux Crustacés des milieux souterrains continentaux et permettent de saisir comment certains groupes d'origine marine ont réussi au cours des temps géologiques leur insertion dans le domaine souterrain par l'intermédiaire de l'interstitiel littoral. Cependant, contrairement à Microcharon, Angeliera, malgré une adaptation profonde à la vie interstitielle eulittorale, comme les Mystacocarides, n'a pas colonisé les nappes phréatiques et demeure inféodé à la frange littorale saumâtre marine.

\section{BIBLIOGRAPHIE}

Ax, P., 1977. Life cycles of interstitial Turbellaria from the eulittoral of the North Sea. Acta zool. 'Fenn., 154: 11-20. Bocquet, $\mathrm{CH}_{\text {., }}$ 1953. Recherches sur le polymorphisme naturel des Jaera marina (Fabr.) (Isopodes Asellotes). Archs. Zool. exp. gén., 90 (4): 187-450. 
Chappuis, P. A., 1942. Eine neue Methode zur Untersuchung der Grundwässerfauna. Act. Sci. Math. Kolozsvar, 6: 3-7.

CoInenu, N., 1971. Les Isopodes interstitiels. Documents sur leur écologie et leur biologie. Mém. Mus. natn. Hist. nat., (n.s.), (A) 64: 1-170.

Coineau, N. \& J. Renaud-Mornant, 1977. Etude anatomique des Isopodes interstitiels. II. Morphologie fonctionnelle et évolution saisonnière de l'appareil génital femelle d'Angeliera phreaticola Chappuis et Delamare Deboutteville 1952. Archs. Zool. exp. gén., 118 (3): 349-366.

Daguerre de Hureaux, N., 1979. Etude du cycle biologique annuel d'une population de Sphaeroma serratum (Isopode, Flabellifère, Sphaeromatidae) du Bassin d'Arcachon. Crustaceana, 37 (1): 85-99.

Delamare Deboutteville, C., 1960. Biologie des eaux souterraines littorales et continentales. Vie Milieu, Suppl. 9: $1-740$.

Faubel, A., 1976. Populations-Dynamik und Lebenszyclen interstitieller Acoela und Macrostomida (Turbellaria). Mikrofauna Meeresboden, 56: 1-107.

Garcin, S. \& J. J. Albaret, 1977. Utilisation de la relation sex ratio/taille pour la décomposition par sexe des structures démographiques. Cah. O.R.S.T.O.M., (Océanogr.) 15 (2): 83-87.

Gine'T, R., 1969. Rythme saisonnier des reproductions de Niphargus (Crustacé Amphipode hypogé). Annls. Spéléol., 24 (2): 87-97.

Hall, J. R. \& R. R. Hessler, 1972. Aspects in the population dynamics of Derocheilocaris typica (Mystacocarida, Crustacea). Vie Milieu, 22 (2A): 305-326.

Hunt, G. W. \& A. Robertson, 1977. The effect of temperature on reproduction of Cyclops vernalis Fisch. (Copepoda, Cyclopoida). Crustaceana, 32 (2): 169-177.

JАKOBI, H., 1954. Biologie, Entwicklungsgeschichte und Systematik von Bathynella natans Vejd. Zool. Jb. (Syst.), 83: (1-2): 1-62.

Janssen, H., M. Scheepmaker, M. van Couwelahr \& S. Pinkster, 1979. Biology and distribution of Gammarus aequicauda and $G$. insensibilis (Crustacea, Amphipoda) in the lagoon system of Bages-Sigean (France). Bijdr. Dierk., 49 (1): 42-70.

JONES, M. B., 1974. Breeding biology and seasonal population changes of Jaera nordmanni nordica Lemercier (Isopoda, Asellota). J. mar. biol. Ass. U.K., 54: 727-736.

JoNES, M. B. \& E. NAYLOR, 1971. Breeding and bionomics of the british members of the Jaera albifrons group of species (Isopoda: Asellota). J. Zool., Lond., 165: 183-199.

Karaman, S., 1933. Neue Isopoden aus unterirdischen Gewässern Jugoslawiens. Zool. Anz., 102: 16-22.

KINNE, O., 1952. Zum Lebenszyclus von Gammarus duebeni Lilj. nebst einigen Bemerkungen zur Biologie von Gammarus zaddachi Sexton subsp. zaddachi Spooner. Veröff. Inst. Meeresforsch. Bremerhaven, 1: 187-203.
Lescher-Moutoué, F., 1974. Sur la biologie et l'écologie des Copépodes Cyclopides hypogés (Crustacés). Annls. Spéléol., 28 (3): 429-502.

Lours, M., 1977. Etude des populations de Talitridae des étangs littoraux méditerranéens. II. Identification des cohortes, cycles et fécondité. Bull. Ecol., 8 (1): 75-86.

Mclachlan, A., 1977. The larval development and population dynamics of Derocheilocaris algoensis (Crustacea, Mystacocarida). Zool. Afric., 12 (1): 1.14.

Magniez, G., 1976a. Contribution à la connaissance de la biologie des Stenasellidae (Crustacea Isopoda Asellota des eaux souterraines). Thèse Doct. ès Sc. Nat., A.O. 10 238, Dijon: 1-228.

—, 1976b. Remarques sur la biologie et l'écologie de Stenasellus virei Dollfus (Crustacea Isopoda Asellota des eaux souterraines). Int. J. Speleol., 8: 135-140.

MARCH, B. G. E. DE, The effects of constant and variable temperatures on the size, growth, and reproduction of the freshwater amphipod Hyalella azteca (Saussure). Can. J. Zool., 56 (8): 1801-1806.

Moceuard, J.-P., G. Besse, P. Juchault, J.-J. Legrand, J. Maissiat, G. Martin \& J.-L. Picaud, 1976. Durée de la période de reproduction chez les femelles de l'Oniscoïde Porcellio dilatatus Brandt suivant les conditions d'élevage: température, photopériode et groupement. Vie Milieu, 26 (1C): 51-76.

,,,,---- -,-- , 1978. Action de la température et de la photopériode sur l'induction des mues parturielles des femelles de Porcellio dilatatus Brandt (Crustacé, Isopode Oniscoïde). Archs. Zool. exp. gén., 119 (2): 409-432.

Mocquard, J.-P., A. Pavèse \& P. Juchault, 1980. Déterminisme de la reproduction saisonnière des femelles d'Armadillium vulgare Latr. (Crustacé, Isopode, Oniscoïde). I. Action de la température et de la photopériode. Annls. Sci. nat., Zool. Biol., (14) 2 (2): 91-98.

Pantoustier, G. \& G. Prunus, 1977. Cycle biologique de l'Isopode Jaera hopeana dans les milieux marins et lagunaires du Nord-Est de la Tunisie. Mar. Biol., 43 (4): $361-367$.

Remane, A., 1927. Halammohydra, ein eigenartiges Hydrozoon der Nord. und Ostsee. Zeitschr. Morph. Ökol. Tiere., 7 (4): 643-677.

-, 1959. Die interstitielle Fauna des Meeressandes. Int. Congr. Zool. London, 15: 320-323.

Renaud-Mornant, J. \& N. Coineau, 1978. Etude anatomique des Isopodes interstitiels. Angeliera phreaticola Chappuis et Delamare, 1952. Données générales. C.R. hebd. Séanc. Acad. Sci., (D) 286 (171): 1249-1252.

Rouch, R., 1968. Contribution à la connaissance des Harpacticides hypogés (Crustacés, Copépodes). Annls. Spéléol., 23 (1): 1-167.

SWRDMARK, B., 1955. Recherches sur la morphologie et la biologie de Psammodrilus balanoglossoides. Archs. Zool. exp. gén., 92: 141-220.

WENNER, A. M., 1972. Sex ratio as a function of size in marine Crustacea. Amer. Natural., 106 (949): 321-350. 